\title{
MOLECULAR CLONING, CHARACTERIZATION AND ENZYMATIC PROPERTIES OF A NOVEL BETA- AGARASE FROM A MARINE ISOLATE PSUDOALTEROMONAS SP. AG52
}

\author{
Chulhong Oh ${ }^{1,3}$, Chamilani Nikapitiya ${ }^{1}$, Youngdeuk Lee ${ }^{1}$, Ilson Whang ${ }^{2}$, Do-Hyung Kang ${ }^{3}$, Soo-Jin Heo ${ }^{3}$, Young-Ung \\ $\mathrm{Choi}^{3}$ and Jehee Lee ${ }^{1,4} *$
}

${ }^{1}$ Department of Marine Life Science, Jeju National University 66 Jejudaehakno, Ara-Dong, Jeju, 690-756, Republic of Korea;

${ }^{2}$ Department of Life Science, Jeju National University 66 Jejudaehakno, Ara-Dong, Jeju, 690-756, Republic of Korea; ${ }^{3}$ Korea Ocean Research \& Development Institute, Ansan, 426-744, Republic of Korea; ${ }^{4}$ Marine and Environmental Institute, Jeju National University, Jeju, 690-814, Republic of Korea.

Submitted: August 04, 2009; Returned to authors for corrections: March 08, 2010; Approved: June 21, 2010.

\begin{abstract}
An agar-degrading Pseudoalteromonas sp. AG52 bacterial strain was identified from the red seaweed Gelidium amansii collected from Jeju Island, Korea. A $\beta$-agarase gene which has $96.8 \%$ nucleotide identity to Aeromonas $\beta$-agarase was cloned from this strain, and was designated as aga $\mathrm{A}$. The coding region is $870 \mathrm{bp}$, encoding 290 amino acids and possesses characteristic features of the glycoside hydrolase family (GHF)-16. The predicted molecular mass of the mature protein was $32 \mathrm{kDa}$. The recombinant $\beta$-agarase (rAgaA) was overexpressed in Escherichia coli and purified as a fusion protein. The optimal temperature and $\mathrm{pH}$ for activity were $55^{\circ} \mathrm{C}$ and 5.5 , respectively. The enzyme had a specific activity of 105.1 and 79.5 unit/mg toward agar and agarose, respectively. The pattern of agar hydrolysis demonstrated that the enzyme is an endo-type $\beta$-agarase, producing neoagarohexaose and neoagarotetraose as the final main products.

Since, Pseudoalteromonas sp. AG52 encodes an agaA gene, which has greater identity to Aeromonas $\beta$ agarase, the enzyme could be considered as novel, with its unique bio chemical characteristics. Altogether, the purified rAgaA has potential for use in industrial applications such as development of cosmetics and pharmaceuticals.
\end{abstract}

Key words: Agar; Aeromonas sp.; $\beta$-agarase; Pseudoalteromonas sp.; GHF-16; Neoagarooligosaccharides

\section{INTRODUCTION}

The main component of the cell wall of marine red algae (Rhodophyceaea) is agar, and it is composed of agarose (4) and agaropectin (12). Agarases are the hydrolytic enzymes mostly found in marine habitats $(5,39)$, which are responsible for the breakdown of agar and agar-derived compounds, and result in oligosaccharides that have various bioactivities $(9,25)$. Based on the pattern of hydrolysis of the substrates, agarases are grouped into $\alpha$-agarases and $\beta$-agarases $(3,19)$. To date, most 
of the agarolytic enzymes isolated, purified and characterized have been from microorganisms, with the majority of the genera being marine bacteria. Among these known agarases, most belong to the $\beta$-agarase group and few biochemical studies have been reported for $\alpha$-agarases $(39,44)$. Several $\beta$ agarases have been purified and characterized from species including Pseudoalteromonas (43, 48), Pseudomonas (16, 17, 33), Alteromonas (19, 24, 29, 39, 49), Agarivorans (15, 20), Vibrio (2, 6, 14, 45), Cytophage (11, 47), Bacillus (46) and Saccharophagus (13). Cloning and expression of $\beta$-agarase encoding genes has been reported from Pseudoalteromonas (30), Pseudomonas (17, 27, 41), Agarivorans (28, 34), Microbulbifer (35-37), Vibrio (45, 50), Zobellia (22) and Streptomyces (23).

Our laboratory is currently conducting research on the characterization of a number of $\beta$-agarases and the biochemical properties of recombinant proteins, from various bacterial species isolated from the marine environment. In this study, Pseudoalteromonas sp. AG52 was isolated from the Jeju Island coastal environment, from which a $\beta$-agarase gene was subsequently isolated and designated as agaA. We cloned the gene, overexpressed the protein in Escherichia coli (E. coli) and purified the recombinant $\beta$-agarase (rAgaA) from Pseudoalteromonas sp. AG52. In addition, purified rAgaA was analyzed for biochemical properties such as specific activities and optimum reaction conditions. Until recently, the Pseudoalteromonas sp. $\beta$-agarase was an unknown entity. Herein we examine and describe in depth this enzyme, which upon analysis showed greater identity to Aeromonas $\beta$-agarase. The characterization of this novel enzyme could be beneficial to a variety of industries.

\section{MATERIALS AND METHODS}

\section{Isolation of agarase-producing bacteria strain}

Agarolytic bacteria were isolated from the red seaweed, Gelidium amansii from the south coast of Jeju Island, Republic of Korea. The crushed seaweeds were spread, on on SWT (0.3\% Tryptone and $1.5 \%$ agar in seawater $)$ SWY $(0.3 \%$ yeast extract and $1.5 \%$ agar in seawater) and marine agar plates (Difco, Detroit, USA). Positive colonies showing clear zones or pits were picked out from the selection plates, and restreaked The pure colonies were selected by repeat streaking under the same conditions and inoculated in their respective broths including $0.2 \%$ agar, then incubated at $30{ }^{\circ} \mathrm{C}$. The stock was prepared from the bacteria culture using $20 \%$ glycerol, then samples were stored at $-70{ }^{\circ} \mathrm{C}$. Polymerase Chain Reaction (PCR) was performed for $16 \mathrm{~S}$ rDNA sequence amplification from the extracted genomic DNA of isolated bacteria. The universal primers $(16 \mathrm{~S}-27 \mathrm{~F}$ as forward and $16 \mathrm{~S}-1492 \mathrm{R}$ as reverse) used for the PCR are shown in Table 1. The sequence was analyzed using the NCBI Blast $\mathrm{N}$ program and the DNAssist program.

\section{Partial agaA gene amplification}

All of the cloning experiments were carried out according to Sambrook et al. (42) with slight modifications. From analyzing the sequences of other agarase genes from NCBI database, three sets of forward and reverse primers were designed (Table 1) and those mix primers (Mix-AGA-F1 and R1, Mix-CY-F2 and R2, Mix-Sa-F3 and R3) were used for initial partial agarase amplification using genomic DNA as template and Ex Taq DNA polymerase (Takara, Japan).

\section{Long and Accurate Polymerase Chain Reaction (LA PCR) for detection of full length agaA}

The complete agaA was cloned using a LA PCR in vitro cloning kit (Takara, Korea), according to the manufacturer instructions. Genomic DNA was digested with separate restriction enzymes BamH I, EcoR I, Hind III and Xho I, and subsequently the digested products were ligated with a $\mathrm{BamH}$ I, EcoR I, Hind III and Xho I cassette, then used as a template for LA PCR. LA52-F1 and LA52-F2 primers and LA52-R1 and LA52-R2 primers were designed to identify the reverse and forward sequence from the known partial sequence of $a g a A$, respectively (Table 1). The amplification of upstream or downstream of the known sequence was performed using LA52-R1 or LA52-F1 with C1 (from the cassette nucleotide 
sequence), respectively. Taking the resultant product as the template, PCR was performed to obtain upstream or downstream of the known sequence using LA52-R2 or LA52F2 with C2 (from the cassette nucleotide sequence), respectively. Product was sequenced, and the gene was analyzed by nucleotide BLAST and Protein BLAST of National Center for Biotechnology Information (NCBI) database. The signal peptide sequence of AgaA was predicted through a SignalP program (http://www.cbs.dtu.dk/services/ SignalP/). The LipoP 1.0 Server (http://www.cbs.dtu.dk/ services/LipoP/) was used for a gram-negative bacteria lipoprotein site search analysis. Identity and percent similarity of full-length amino acid sequences were calculated using FASTA program (38).

Table 1. Oligonucleotide primers

\begin{tabular}{lll}
\hline Name & Object & Sequence (5' to 3' direction) \\
\hline 16S-27F & 16S rDNA sequence amplification & AGAGTTTGATCMTGGCTCAG \\
16S-1492R & 16S rDNA sequence amplification & TACGGYTACCTTGTTACGACTT \\
Mix-AGA-F1 & agaA partial sequence amplification & CWTCKTATATWAATGCTTGGC \\
Mix-AGA-R1 & agaA partial sequence amplification & TGGYTGRTAATCTTGAAATGG \\
Mix-CY-F2 & agaA partial sequence amplification & YTNGARTAYTAYATHGAYGG \\
Mix-CY-R2 & agaA partial sequence amplification & TTRTANACNCKDATCCARTC \\
Mix-Sa-F3 & agaA partial sequence amplification & TCNATHCAYYTNTAYGAYTTYCC \\
Mix-Sa-R3 & agaA partial sequence amplification & CCAYTCNGCYTTNACNGG \\
52LA-F1 & LA PCR Forward 1 & TCGTCGCTACGGTGTTCATTGGAA \\
52LA-F2 & LA PCR Forward 2 & TAGTTCGCAGCGTTTCAGGTCCTA \\
52LA-R1 & LA PCR Reverse 1 & ACGTGCATACGTTGGTCAAACCAC \\
52LA-R2 & LA PCR Reverse 2 & TGCCTCCATCGCATCAATTTCCTG \\
C1 & Cassette primers & GTACATATTGTCGTTAGAACGCGTAATACGACTCA \\
C2 & Cassette primers & CGTTAGAACGCGTAATACGACTCACTATAGGGAGA \\
Ag52-F1 & Cloning to pET-16b & (GA) ${ }_{3}$ CATATGGCAGATTGGGACGCATATAGTA $($ Nde I) \\
Ag52-R2 & Cloning to pET-16b & (GA) ${ }_{3}$ GGATCCTTAGTTTGCTTTGTAGACACGTATC $($ BamH I) \\
\hline
\end{tabular}

Cloning of AgaA coding sequence into the expression vectors

Primer set Ag52-F1 and Ag52-R2 were designed with its corresponding restriction enzyme sites (Table 1) to clone the coding sequence into the pET16b expression vector (Novagen, USA), without including its signal sequence. The vector and PCR products were digested with restriction enzymes, ligated and transformed into $E$. coli DH5 $\alpha$ cells, and correct recombinants (confirmed by restriction enzyme digestion and sequencing) were transformed into E. coli BL21(DE3). Recombinant cells were overexpressed in the presence of isopropyl- $\beta$-thiogalactopyranoside (IPTG) at a final concentration of $1 \mathrm{mM}$. Briefly, $5 \mathrm{~mL}$ of an overnight grown BL21(DE3) starter culture was inoculated into $100 \mathrm{~mL}$ Luria broth with $100 \mu \mathrm{L}$ ampicillin $(100 \mathrm{mg} / \mathrm{mL})$ and $10 \mathrm{mM}$ glucose $\left(0.2 \%\right.$ final concentration) and incubated at $37{ }^{\circ} \mathrm{C}$. Recombinant pET-16b- AgaA cells were induced for $24 \mathrm{~h}$ at 12 ${ }^{\circ} \mathrm{C}$, followed by cell harvesting (centrifugation at $4000 \mathrm{x} \mathrm{g}$ for $20 \mathrm{~min}$ at $4{ }^{\circ} \mathrm{C}$ ). The cells carrying the pET-16b- AgaA were resuspended in $5 \mathrm{~mL}$ ice-cold $1 \mathrm{x}$ binding buffer $(8 \mathrm{x}=4 \mathrm{M}$ $\mathrm{NaCl}, 160 \mathrm{mM}$ Tris $\mathrm{HCl}, 40 \mathrm{mM}$ imidazole, $\mathrm{pH}$ 7.9) and frozen at $-20{ }^{\circ} \mathrm{C}$ overnight. After thawing on ice, the bacterial cells 
were sonicated and supernatant was taken as a crude enzyme after centrifugation. The crude rAgaA fusion protein fused with His tag was purified using the His Bind purification Kit (Novagen, USA). Elutes were collected in $500 \mu \mathrm{L}$ fractions and respective elutes were run on $12 \%$ SDS-PAGE. The concentrations of purified proteins were determined by the method of Bradford using bovine serum albumin (BSA) as the standard.

\section{Agarase enzyme assay}

Specific activity of purified rAgaA was determined according to a modified method of Ohta et al. (35) using different substrates including $1 \%$ food-grade agar, $1 \%$ agarose and $1 \%$ carrageenan. Appropriately diluted enzyme solution was added to different substrates in phosphate buffer $(\mathrm{pH} 7.0)$ at $45{ }^{\circ} \mathrm{C}$, and was incubated at $45{ }^{\circ} \mathrm{C}$ for $30 \mathrm{~min}$. Activity was expressed as the initial rate of agar hydrolysis by measuring the release of reducing ends using the 3,5-dinitrosalicylic acid (DNS) procedure (32) with D-galactose as the standard. One unit of the enzyme activity was defined as the amount of protein per min produced $1 \mu \mathrm{mol}$ of reducing sugar as Dgalactose under condition of the assay.

\section{Biochemical characterization of rAgaA}

In each experiment, $1 \%$ agar solution and purified agarase were mixed and incubated at various times and temperatures as described. The relative agarase activity was determined by the DNS method. The optimum temperature of $r$ AgaA activity was determined by monitoring the relative enzymatic activity at temperatures ranging from $40-65{ }^{\circ} \mathrm{C}$ with $5{ }^{\circ} \mathrm{C}$ intervals at $\mathrm{pH}$ 7.0. Optimum $\mathrm{pH}$ was tested from $\mathrm{pH}$ 4.5-9.0 with $\mathrm{pH} 0.5$ intervals at $45{ }^{\circ} \mathrm{C}$. Acetate buffer and phosphate buffer were used for $\mathrm{pH}$ 4.5-6.0 and $\mathrm{pH}$ 6.5-9.0, respectively. The thermostability of $\mathrm{rAgaA}$ was evaluated by measuring the residual activity of the enzyme after incubation at the temperatures between $40-55{ }^{\circ} \mathrm{C}$ for 30,60 and $120 \mathrm{~min}$. The effects of various metal ion salts and chelators on purified $r$ AgaA activity were tested by determining the activity in the presence of $2 \mathrm{mM}$ of various ions or chelators $\left(\mathrm{CaCl}_{2}, \mathrm{CuSO}_{4}\right.$,
$\mathrm{FeSO}_{4}, \mathrm{KCl}, \mathrm{MgSO}_{4}, \mathrm{MnCl}_{2}, \mathrm{NaCl}$ and EDTA) in a final concentration and incubated at $45{ }^{\circ} \mathrm{C}$ for $30 \mathrm{~min}$. The control was the assay mixture, with no addition of metal ion salts or chelators.

\section{Identification of rAgaA hydrolyzed agar products}

Thin layer chromatography (TLC) was used to identify the hydrolysis products of agar and neoagarooligosaccharides. Neoagarohexanitol (NA6) was purchased from Sigma (USA) and neoagarotetraose (NA4) and neoagarobiose (NA2) (NA4 + NA2) were prepared by digestion of neoagarohexanitol using commercial $\beta$-agarase (New England Biolab, USA). D-(+)galactose was purchased from Sigma (USA), and all above mentioned oligosaccharides were used as standards. Moreover, food-grade agar and NA6 were used as substrates for the reactions. The reaction of purified agarase and agar was carried out in $200 \mu \mathrm{L}$ reactions containing $20 \mu \mathrm{L}$ of purified agarase and $180 \mu \mathrm{L}$ of $1 \%$ agar at $45{ }^{\circ} \mathrm{C}$ for 30,60 , and $120 \mathrm{~min}$. The NA6 substrate was incubated separately with $20 \mu \mathrm{L}$ of purified agarase at $45{ }^{\circ} \mathrm{C}$ for $120 \mathrm{~min}$. Subsequently, the reaction mixtures were applied to a silica gel 60 TLC plate (Merck, Germany). The TLC plates were developed using a solvent system consisting of $n$-butanol: acetic acid: water $(2: 1: 1, v / v)$. After hydrolysis of substrates, the resultant oligosaccharide spots were visualized by spraying $10 \% \mathrm{H}_{2} \mathrm{SO}_{4}$ on the plate, then heating it on a hot plate.

\section{Nucleotide sequence accession number}

The Pseudoalteromonas sp. AG52 $\beta$-agarase nucleotide sequence was submitted to the NCBI database with accession number FJ979637.

\section{RESULTS}

\section{Identification of the agarase-producing bacteria}

Initially, agarase-producing marine bacteria were isolated from selection plates showing clear zones. The 16S rRNA sequence analyses results showed that the isolated bacteria from seaweed was $99 \%$ similar to Pseudoalteromonas sp. and 
is assigned to the genus Pseudoalteromonas and named Pseudoalteromonas sp. AG52. This strain was deposited in Korean Culture Collection Center of Microorganisms (KCCM), Rep. of Korea with accession number KCCM 42924.

\section{Cloning of $\beta$-agarase from Pseudoalteromonas sp AG52}

The partial agaA sequence $(651 \mathrm{bp})$ followed by full length sequence was amplified as described in Materials and Methods. The nucleotide and deduced amino acid sequences of Pseudoalteromonas sp. AG52 $\beta$-agarase is shown in Figure 1. The aga $\mathrm{A}$ gene open reading frame (ORF) consists of a $870 \mathrm{bp}$ encoding a protein of 290 amino acids. The agaA has a putative molecular mass of $32 \mathrm{kDa}$ with an isoelectric point of 5.8. The signal peptide (1-21 aa) and lipoprotein signal peptide (signal peptidase II) were identified in the $\mathrm{N}$-terminal sequence. Additionally, a characteristic GHF-16 $\beta$-agarase family domain $\left(\mathrm{Cys}^{22}\right.$-Lys $\left.{ }^{287}\right)$, catalytically active site residues $\left(\mathrm{Tyr}^{69}, \mathrm{Asp}^{71}, \operatorname{Trp}^{72}, \operatorname{Trp}^{139}, \mathrm{Ser}^{145}, \mathrm{Asp}^{150}, \mathrm{Glu}^{153}, \mathrm{Phe}^{176}\right.$, $\left.\mathrm{Arg}^{178}, \mathrm{Glu}^{257}, \mathrm{Glu}^{259}\right)$, and calcium binding residues $\left(\mathrm{Gln}^{47}\right.$, $\mathrm{Phe}^{48}, \mathrm{Asn}^{49}, \mathrm{Gly}^{91}, \mathrm{Ala}^{92}, \mathrm{Asp}^{282}, \mathrm{Trp}^{283}$ ) were identified in the sequence. The agaA nucleotide sequence showed $96.4 \%$ and $74.9 \%$ nucleotide identity to $\beta$-agarase sequence of Aeromonas sp. (Accession number U61972) and Pseudoalteromonas atlantica (accession number M73783), respectively. Database searches using BLASTP yielded results showing homology to other known GHF-16 family agarases. Pairwise comparison of AgaA amino acid sequence to known agarases is shown in Table 2. Interestingly, AgaA amino acid sequence shares 96.9\% identity and $99.3 \%$ similarity with Aeromonas sp. $\beta$ agarase (accession number AAF03246). The $P$. atlantica $\beta$ agarase coding sequence (accession number AAA91888) shares only $84.5 \%$ identity, and $92.4 \%$ similarity with AgaA. The multiple alignment shows conserved catalytic and calcium binding residues of AG52 when compared with other GHF-16 agarases (Figure 2). The phylogenetic analysis of the AgaA amino acid sequence including known $\beta$-agarase amino acid sequences of different members of the $\beta$-agarase family (GHF16, 52 and 82) was constructed using the neighborjoining method as shown in Figure 3. The AgaA coding sequence was clustered with GHF-16 $\beta$-agarases, and formed a monophyletic clade with $\beta$-agarase of Aeromonas sp. and $P$. atlantica (AAA91888). However, it was also closely grouped with Aeromonas $\beta$-agarase.

\section{Purification and Biochemical characterization of rAgaA}

Successfully purified rAgaA agarase in pET-16b as a fusion protein was approximately $33 \mathrm{kDa}$, which was in agreement with the predicted molecular mass of AgaA (Figure 4), hence enzyme characterization was carried out with the fusion protein.

The specific activity of purified rAgaA towards agar and agarose were 105.1 and 79.5 unit/mg, respectively. The optimum reaction temperature of the purified rAG52 was $55{ }^{\circ} \mathrm{C}$ (Figure 5A), however, more than $50 \%$ of the relative activity was observed between 40 and $60{ }^{\circ} \mathrm{C}$. The effect of $\mathrm{pH}$ on purified enzyme is shown in Figure 5B. The maximal activity was observed at pH 5.5 and the enzyme was stable (more than $60 \%$ relative activity) in the range of buffers from $\mathrm{pH} 4.5-9$ under the conditions of the assay. The temperature dependence of the rAgaA activity on agar was determined by measuring the activity at various temperatures for $120 \mathrm{~min}$. The effect of temperature on the stability of rAG52 is shown in Figure 5C. The thermostability of rAgaA was retained upto $80 \%$ at $40{ }^{\circ} \mathrm{C}$ for $30 \mathrm{~min}$. When temperature was increased to 45,50 and 55 ${ }^{\circ} \mathrm{C}$ for $30 \mathrm{~min}$, the enzyme relative activity was decreased to $20-30 \%$. Nevertheless, the enzyme was fairly stable $(50 \%$ relative activity) at $40{ }^{\circ} \mathrm{C}$ for $60 \mathrm{~min}$ of incubation. Moreover, addition of $2 \mathrm{mM} \mathrm{CaCl}$ to the reaction mixture at $40{ }^{\circ} \mathrm{C}$ was enhanced the thermostability by 20 and $15 \%$ at 60 and 120 min, respectively (Figure 5D). The effect of metal ion salts and chelators on the activity of purified rAgaA enzyme is shown in Figure 6. rAgaA relative activity was totally inhibited by divalent metal salts such as $\mathrm{CuSO}_{4}$ and $\mathrm{ZnSO}_{4}$ (each at $2 \mathrm{mM}$ final concentration). Moreover, $2 \mathrm{mM}$ EDTA inhibited the enzyme activity by $60 \%$. In contrast, $2 \mathrm{mM} \mathrm{FeSO}_{4}$ and $2 \mathrm{mM}$ $\mathrm{KCl}$ enhanced the activity of $\mathrm{rAgaA}$ more than the other metal ion salts present in seawater. 


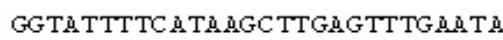

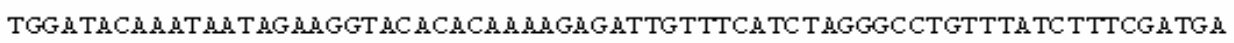

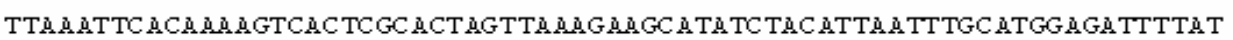

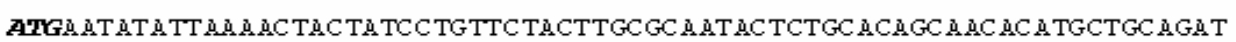

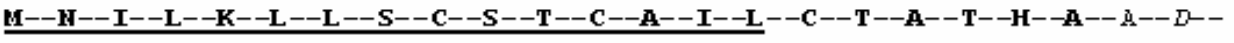
TGGGACGCATATAGTA TTCCGGCTTCTGCTGGA TCAGGT MA HACATGCA TTACAMACTGTTTCCGAC

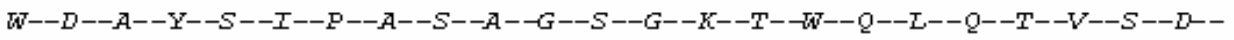
28 97 166 235

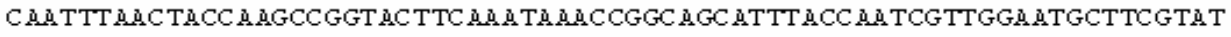

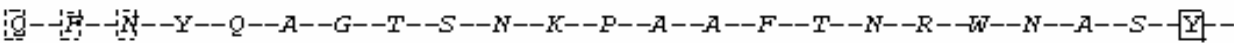
69

ATTAATGCTTGGCTTGGGCCTGGTGATACTGÄ TTCAGTTCAGGTCATTCCTACACTÄCTGGTGGTGCG 442

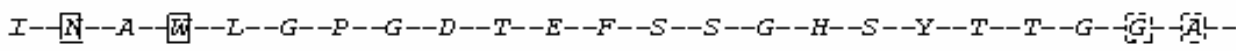
92

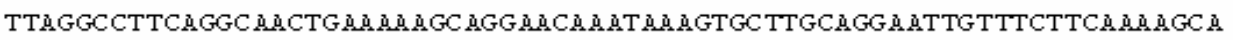
511

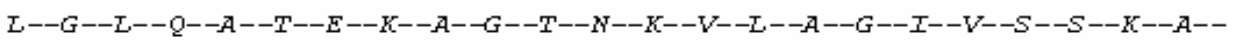
115

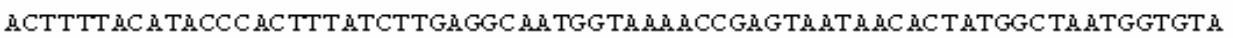
580

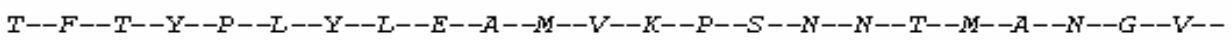
138 TGGATGTTGAGCTCTGATTCAACTCAGGALATTGATGCGATGGAGGCATACGGCAGTGATCGTGTAGGG 649

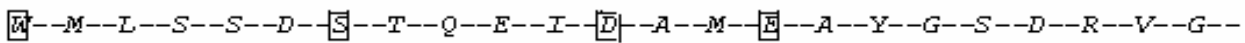
161

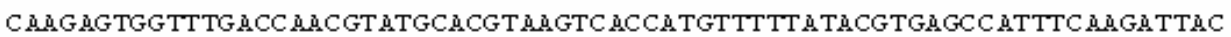
718

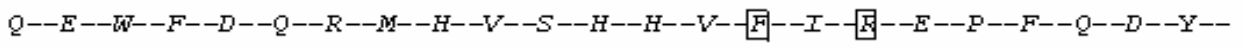
184

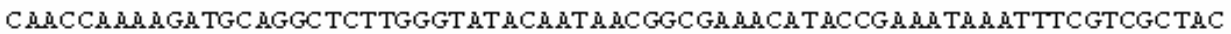
787

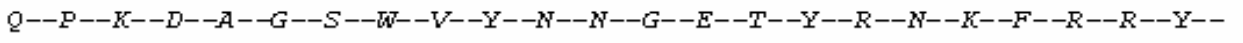
207 GGTGTTCATTGGÄGGACGCGTGGḦCCTAGATTACTATATTGATGGTGTATTAGTTCGCAGCGTTTCA 8.56 $G--V--H-D T--K--D--A-D-W--N--I--D--Y--Y--I-D-D-G--V--I--V--R--S--V--S--$ $23 \square$

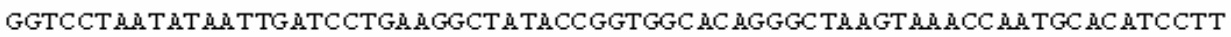
925

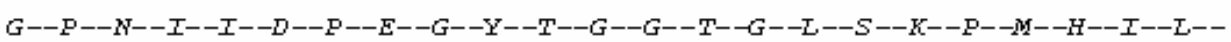
2.53

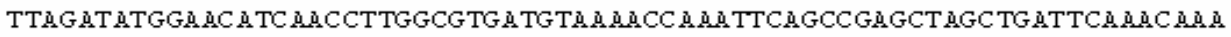
994

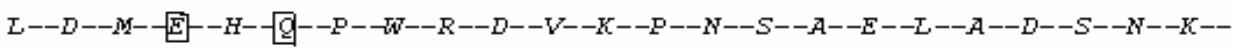
276

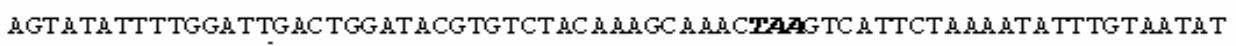
1063 S-ーI--F-ーW--I290

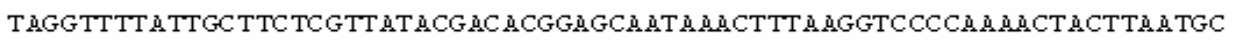
1132 GGCTATTACAGCCGCATT ÄGTAT ÄTTÄCCTGÄCTCTGGAT AGTÄÄTCTATCTCGAGCAGCTATT $12 \square 1$ GACGCGTGÄTTCTCTCCCTÄTÄGTG ÄTCGTÁ

Figure 1. The nucleotide and deduced amino acid sequences of the $\beta$-agarase of Pseudoalteromonas sp. AG52. The predicted lipoprotein signal peptide is underlined and signal peptide sequence is in bold face. The start (ATG) and stop (TAA) codons are in bold italics and stop codon is marked with an asterisk (*). The GHF-16 $\beta$-agarase domain is in italics. Active sites and calcium binding residues are in boxes and dotted boxes, respectively. 
Table 2. Pairwise analysis and comparisons of the deduced amino acid sequence of Pseudoalteromonas sp. AG52 $\beta$-agarase with other known $\beta$-agarases.

\begin{tabular}{llll}
\hline Species & Accession number & Identity (\%) & Amino acids \\
\hline Aeromonas sp. $\beta$-agarase & AAF03246 & 96.9 & 290 \\
Pseudoalteromonas atlantica $\beta$-agarase & AAA91888 & 84.5 & 290 \\
AguD uncultured bacterium $\beta$-agarase & AAP49316 & 45.2 & 449 \\
Zobellia galactanivorans $\beta$-agarase B & AAF21821 & 39.3 & 353 \\
Pseudomonas sp. ND137 agarase & BAB79291 & 38.7 & 441 \\
Microbulbifer thermotolerans agarase & BAD29947 & 36.1 & 433 \\
Microbulbifer elongatus agarase & BAC99022 & 35.6 & 441 \\
Cellvibrio sp. OA-2007 putative agarase & BAH16616 & 32.3 & 596 \\
Saccharophagus degradans $\beta$-agarase I & AAT67062 & 26.8 & 593 \\
Zobellia galactanivorans $\beta$-agarase A & AAF21820 & 26.0 & 539 \\
Pseudoalteromonas sp. CY24 agarase & AAN39119 & 24.1 & 453 \\
\hline
\end{tabular}

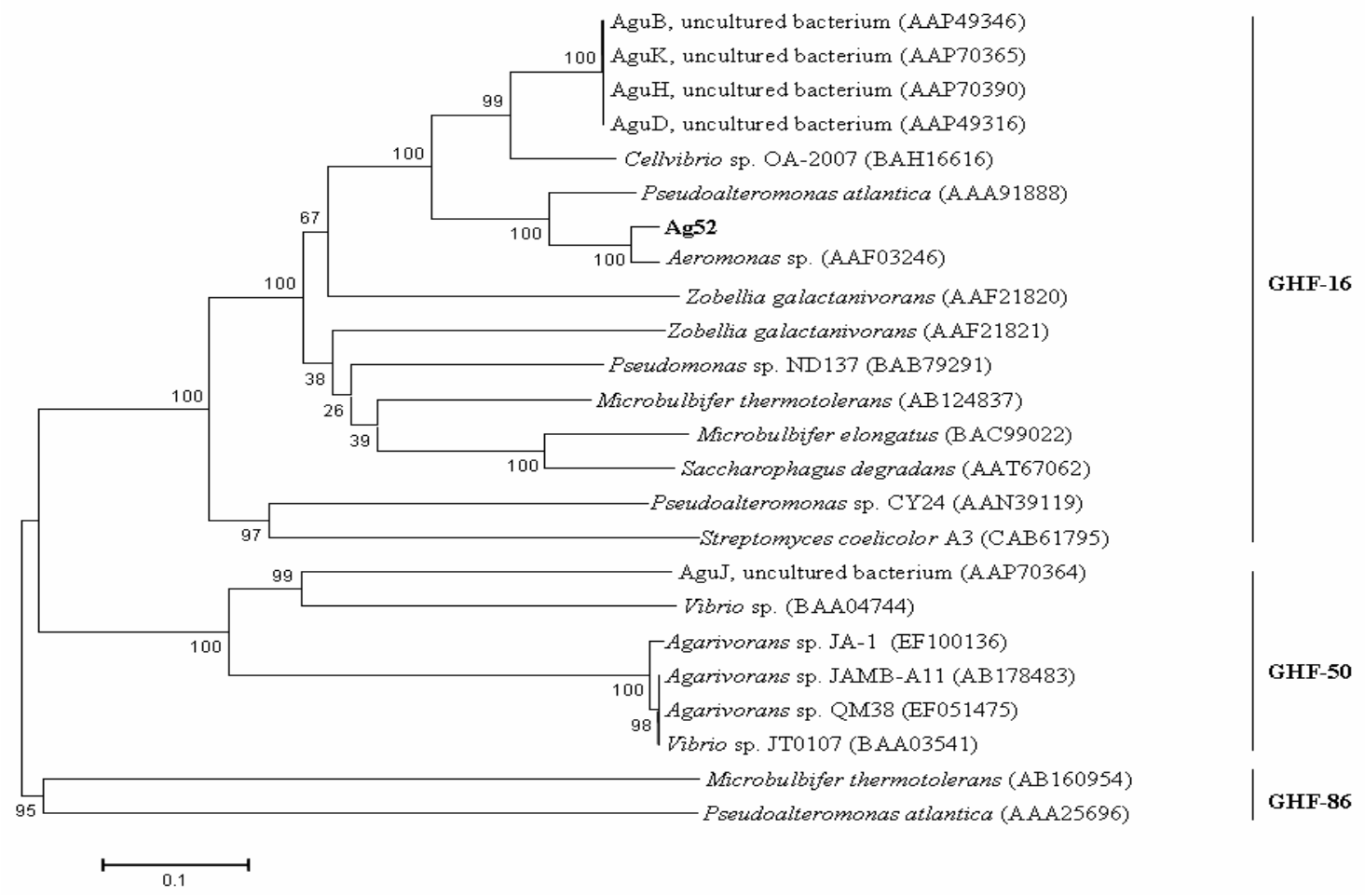

Figure 3. Phylogenetic analysis of AgaA with known agarases based on amino acid sequence. Phylogenetic analysis was done by the Neighbor Joining method using MEGA3.1, based on sequence alignment using ClustalW (1.81). Numbers indicate the bootstrap confidence values of 1000 replicates. The accession numbers of the selected agarase sequences are as follows: AB178483, agarase (Agarivorans sp. JAMB-A11); EF051475, QM38 agarase (Agarivorans sp. QM38); EF100136, $\beta$-agarase (Agarivorans sp. JA-1); AAA25696, $\beta$-agarase precursor (Pseudoalteromonas atlantica); AAP49346, AguB; AAP70390, AguH; AAP70365, AguK; AAP49316, AguD from uncultured bacterium; AAA91888, $\beta$-agarase I (Pseudoalteromonas atlantica); AAF03246, $\beta$-agarase (Aeromonas sp.); AB124837, agarase (Microbulbifer thermotolerans); BAC99022, agarase (Microbulbifer elongatus); BAB79291, agarase, (Pseudomonas sp. ND137; AAF21821, $\beta$-agarase B precursor (Zobellia galactanivorans); AAF21820, ß-agarase A precursor (Zobellia galactanivorans); AAN39119, extracellular agarase precursor, (Pseudoalteromonas sp. CY24); CAB61795, extracellular agarase precursor (Streptomyces coelicolor A3); AAP70364, AguJ (uncultured bacterium); BAA04744, $\beta$-agarase (Vibrio sp.); BAA03541, $\beta$-agarase (Vibrio sp. JT0107); BAH16616, agarase (Cellvibrio sp. OA2007); AAT67062, $\beta$-agarase I (Saccharophagus degradans); AB160954, $\beta$-agarase (Microbulbifer thermotolerans). 

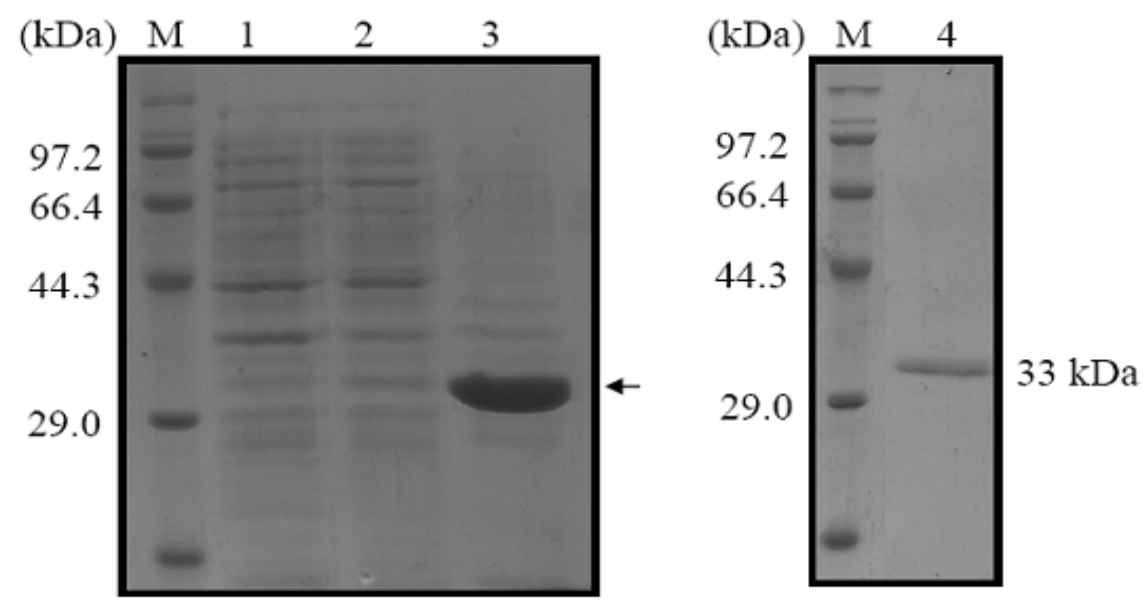

Figure 4. SDS-PAGE of the rAgaA. Samples of rAgaA were separated on 12\% SDS-PAGE and stained with Coomassie brilliant blue. M: molecular mass marker (BioRad, USA). Lane 1: total cellular extract from E. coli BL21 (DE3) before induction; lane 2: total cellular soluble extract after induction; lane 3: total cellular insoluble extract after induction; lane 4: purified rAgaA.
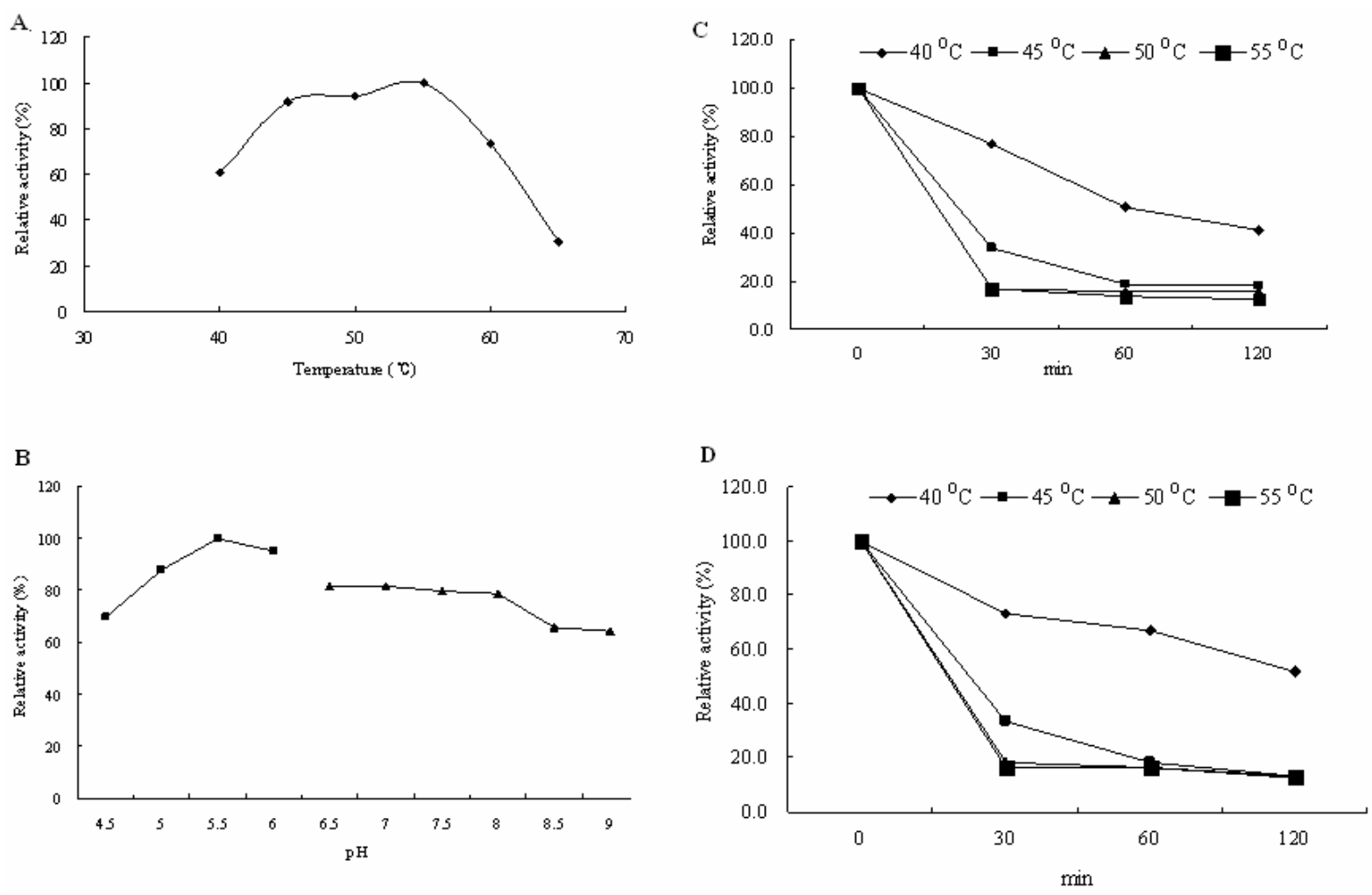

Figure 5. Characterization of biochemical properties of purified rAgaA. A. The effect of temperature on the rAgaA. The effect of temperature on enzyme activity was determined under standard assay conditions as described in Materials and Methods, at temperatures ranging between 40-65 ${ }^{\circ} \mathrm{C}$. B. The effect of $\mathrm{pH}$ on the activity of rAgaA. Optimum $\mathrm{pH}$ for rAgaA activity was examined from $\mathrm{pH} 4.5-9.0$ at $\mathrm{pH} 0.5$ intervals at $45{ }^{\circ} \mathrm{C}$ under standard assay conditions (described in Materials and Methods) using acetate ( $\mathrm{pH}$ 4.5-6.0) and phosphate buffer ( $\mathrm{pH}$ 6.5-9.0). C. The effect of thermostability on rAgaA at different temperatures at different time points. Thermostability was determined by measurement of residual activity under standard assay conditions as described in Materials and Methods at temperatures between $40-55^{\circ} \mathrm{C}$ for 30,60 and 120 min. D. The effect of $2 \mathrm{mM} \mathrm{CaCl}_{2}$ on thermostability of rAgaA. 


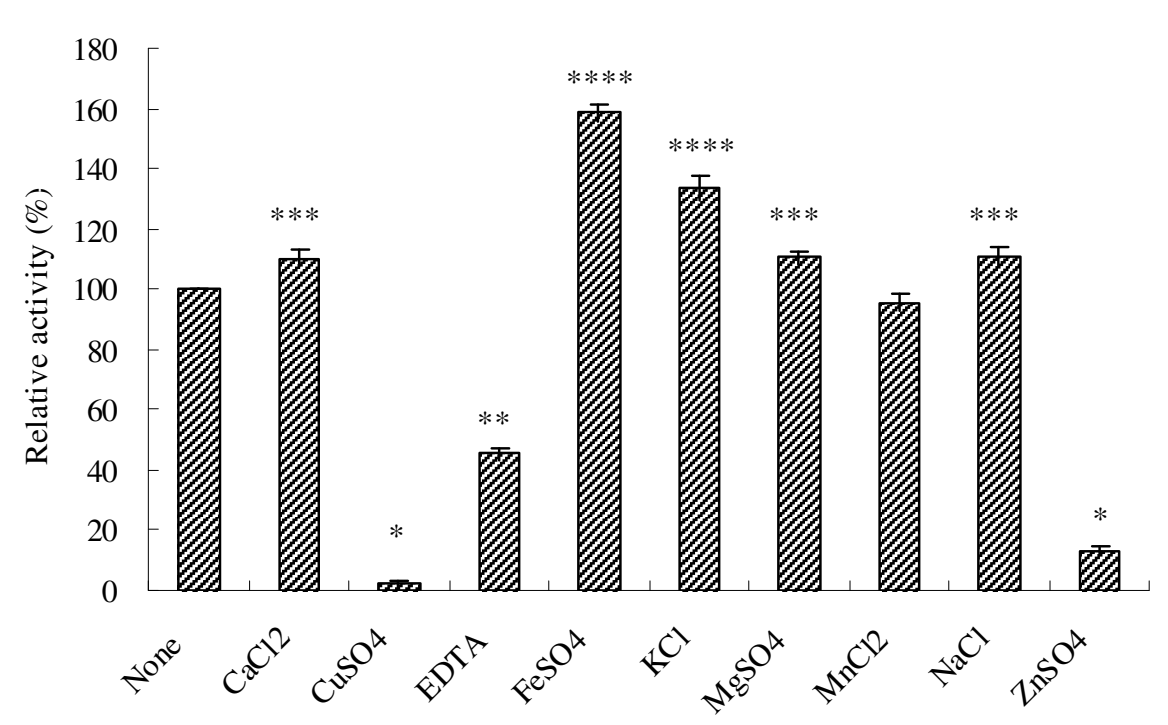

Figure 6. The effects of metal ions and metal salts on the activity of purified $\mathrm{rAgaA}$. Various ions or chelators $\left(\mathrm{CaCl}_{2}, \mathrm{CuSO}_{4}\right.$, $\mathrm{FeSO}_{4}, \mathrm{KCl}, \mathrm{MgSO}_{4}, \mathrm{MnCl}_{2}, \mathrm{NaCl}$ and EDTA) at a final concentration of $2 \mathrm{mM}$ were included in the reaction buffer to test the activity of $\mathrm{rAgaA}$ at $45^{\circ} \mathrm{C}$ for $30 \mathrm{~min}$. The data presented are the average of three replicates. Means with the same number of stars are not significantly different at $\mathrm{p}<0.05$, based on ANOVA. Error bars represent \pm SD.

\section{Identification of hydrolysis products of the rAgaA by TLC}

Hydrolysis patterns of the purified rAgaA against foodgrade agar and neoagarohexanitol (NA6) are shown in Figure 7. When rAgaA was incubated with agar, two distinct spots namely, NA6 and NA4 were observed on TLC plates at 30, 60 and $120 \mathrm{~min}$ after the reaction. Furthermore, the distinct spot of NA4 was observed when rAgaA was incubated with NA6.

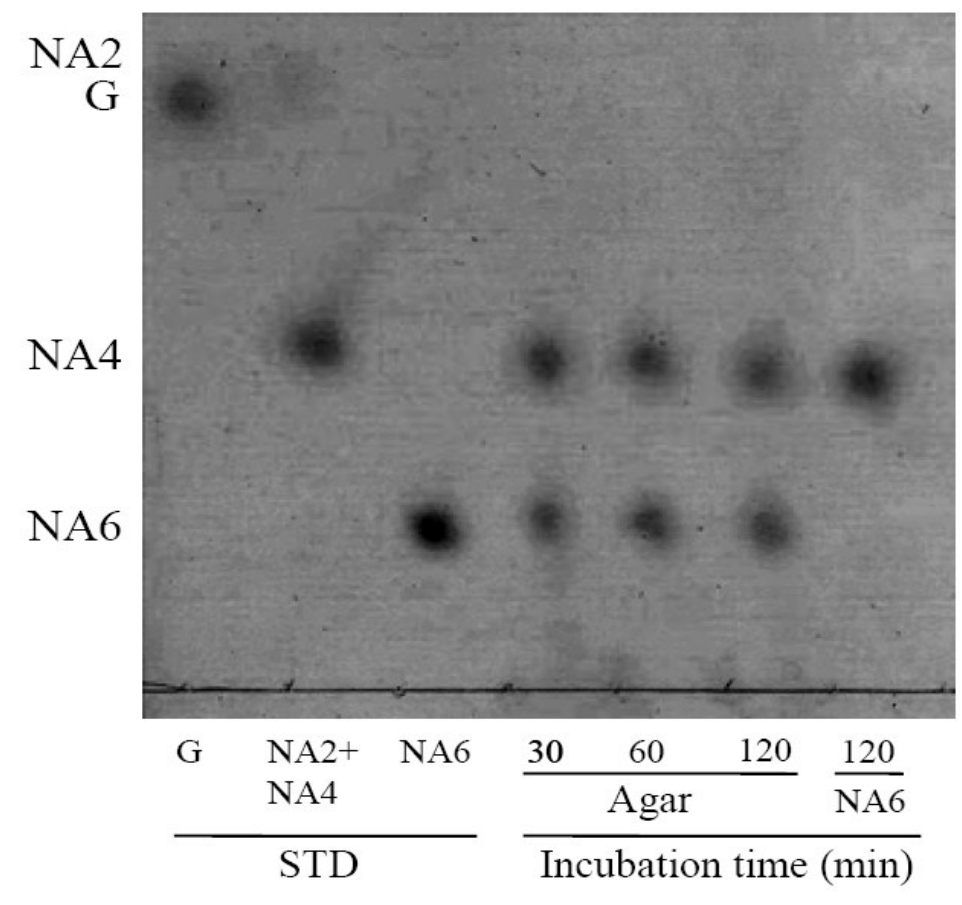

Figure 7. TLC of hydrolysis products of the purified rAgaA on food-grade agar and neoagarooligosaccharides. The assay of rAgaA and agar were performed in $200 \mu \mathrm{l}$ reactions containing 20 $\mu \mathrm{L}$ of purified agarase and $180 \mu \mathrm{L}$ of $1 \%$ agar at 45 ${ }^{\circ} \mathrm{C}$ for 30,60 , and $120 \mathrm{~min}$. The NA6 substrate was incubated with $20 \mu \mathrm{l}$ of $\mathrm{rAgaA}$ separately at $45^{\circ} \mathrm{C}$ for $120 \mathrm{~min}$. Neoagarohexaitol (NA6), neoagarotetraose (NA4), neoagarobiose (NA2) and D-(+)-galactose (G) were used as standards (STD). 


\section{DISCUSSION}

In this study, a newly found marine bacterial isolate was assigned to the genus Pseudoalteromonas based on the $16 \mathrm{~S}$ rDNA sequence analysis. We report herein the cloning and sequencing of the $\beta$-agarase gene with $\beta$-agarase activity from Pseudoalteromonas sp. AG52, which has greater identity to the Aeromonas $\beta$-agarase coding sequence than the Pseudoalteromonas sp. $\beta$-agarase coding sequence in the NCBI Genbank. It was shown in this study that $a g a \mathrm{~A}$ was genetically closely related to the GHF-16 $\beta$-agarases, and hence this gene should be classified into the GHF-16 family. The primary structure of the Pseudoalteromonas sp. AG52 $\beta$-agarase shows regions homologous to GHF-16 family members, which include catalytic domains belonging to GHF-16, which hydrolyze the internal $\beta$-1,4-linkage of agar, producing neoagarooligosaccharides. BLASTP and pairwise analysis of $a g a \mathrm{~A}$ full length coding sequence with other agarases which belong to the GHF-16 family showed overall sequence identities ranging from $24-97 \%$. Some of the $\beta$-agarases encode for a modular protein consisting of a signal peptide, catalytic module and C-terminal domain of unknown function or a carbohydrate binding module $(1,35)$, and this may be the reason for having low identity, even within the same family. It has been reported that homologous regions in most of the reported GHF-16 agarases were present in catalytic module of the $\beta$-agarases (1). This further confirms the heterogeneity of the amino acid sequences in length, catalytic properties and substrate specificities in agarases. Furthermore, the results of the phylogenetic analysis support the idea that many $\beta$-agarases may have evolved from a common ancestral form, and, that domain shuffling may contribute significantly to the diversity of the agarases $(7,10)$. Even though the protein is divergent from the primary sequences, AgaA features strictly conserved catalytic residues, which show conservation among the GHF16 family members. Glutamic and aspartic acid are the highly conserved active site residues, which are responsible for catalytic activity in the GHF (30). According to previous reports $(1,31)$, the conserved $\mathrm{Glu}^{148}$ and $\mathrm{Glu}^{153}$ are responsible for acting as the nucleophile and the acidic/basic residues, respectively, in AgaA. The third conserved acidic residue at $\mathrm{Asp}^{150}$, is responsible for maintaining the charges in the environment of catalytic amino acids. The lipoprotein signal peptide is one of the secretion systems reported for gram negative bacteria (40) and such a signal was predicted in the AgaA sequence at the $\mathrm{N}$-terminal where $\mathrm{Cys}^{16}$ may be linked to the lipid moiety. However, in this study, the cleavage of $\mathrm{N}$ terminal hydrophobic segment is by either signal peptidase I or signal peptidase II is not known clearly and remains to be elucidated. Using pET16b, intracellular AgaA was expressed efficiently and purified as a fusion protein. The purified agarase had a molecular mass of $33 \mathrm{kDa}$, which is close to those reported for $\beta$-agarases from Pseudoalteromonas sp. N-1 (33 kDa) (48) and Pseudomonas atlantica (32 kDa) (33); but, smaller than agarases reported for Alteromonas sp. (52 kDa) (29) and Pseudomonas sp. W7 (59 kDa) (17); and, larger than agarases reported for Vibrio sp. AP-2 (20kDa) (2). AgaA hydrolyzes agar to give NA6 and NA4 as the main products, suggesting that the enzyme is a $\beta$-agarase, in accordance with most of the reported GHF-16 $\beta$-agarases $(1,43)$.

Generally, most of the GHF-16 $\beta$-agarases that have been characterized so far, have optimal function at $40{ }^{\circ} \mathrm{C}$ and $\mathrm{pH}$ optimums in the range of neutral to mild alkaline (50). rAgaA has an optimum temperature $\left(55{ }^{\circ} \mathrm{C}\right)$ that is higher than the gelling temperature of agar $\left(40{ }^{\circ} \mathrm{C}\right)$ with broad range of $\mathrm{pH}$. These are properties which are useful for the production of industrially important oligosaccharides from marine algae or agar. These findings are in contrast with earlier reported $\mathrm{pH}$ and temperature optima at 7 and $30{ }^{\circ} \mathrm{C}$, respectively, for a purified $\beta$-agarase from Pseudoalteromonas sp. (48). A temperature optimum at $40{ }^{\circ} \mathrm{C}$ and a $\mathrm{pH}$ optimum of 6.0 have been reported for recombinant $\beta$-agarase AgaB from Pseudoalteromonas sp. CY24 (30). In contrast to the experimentally determined optimum temperature of $\mathrm{rAgaA}$ activity, thermal stability profiles of this protein showed that the enzyme is stable under $45^{\circ} \mathrm{C}$ even after $30 \mathrm{~min}$ incubation, however, it becomes inactivated at higher temperatures. The enzyme at $40{ }^{\circ} \mathrm{C}$ retained $50 \%$ activity even after $1 \mathrm{~h}$ of 
incubation, indicating that this enzyme might be used under mild heating conditions. In addition, the enzyme stability was considerably enhanced in the presence of $\mathrm{CaCl}_{2}$, and enzyme retained more than $15 \%$ of its initial activity after 1 and $2 \mathrm{~h}$ of incubation with $\mathrm{CaCl}_{2}$. Several published studies support these results, where a major role is proposed for $\mathrm{Ca}^{2+}$ in stabilization of enzymes at higher temperatures $(18,21)$. It was reported that the reason for this may be due to the strengthening of interactions inside the protein molecule, and probably by the binding of $\mathrm{Ca}^{2+}$ to an autolysis site $(8,26)$. It has been reported that a catalytic module of $\beta$-agarase ( $\beta$-AgaA_CM) of $Z$. galactetanivorans Dsij, had $\mathrm{Ca}^{2+}$ bound on the convex face of the protein in an octahedral geometry, coordinating with the backbone carbonyl oxygen atoms of $\mathrm{Ser}^{47}, \mathrm{Ser}^{91}$, and $\mathrm{Asp}^{279}$, a carboxylate oxygen of $\mathrm{Asp}^{279}, \mathrm{Asn}^{49}$, and $\mathrm{Asp}^{22}$, and one water molecule. In the $\beta$-agarase $(\beta$-AgaB) of $Z$. galactetanivorans Dsij, the $\mathrm{Ca}^{2+}$ was positioned in the same locations in a pentahedral manner to the backbone carbonyl oxygen atom of $\mathrm{Asn}^{83}, \mathrm{Gly}^{127}$, and $\mathrm{Asp}^{343}$, a carboxylate oxygen of $\mathrm{Asp}^{343}$, and one water molecule (1). Interestingly, in AG52, the $\mathrm{Ca}^{2+}$ binding residues were located at the same positions at $\mathrm{Gln}^{47}$, Gly $^{91}$ and $\mathrm{Asp}^{282}$, however, the precise geometrical arrangement of $\mathrm{Ca}^{2+}$ binding needs to be investigated in future. In addition, analysis of the amino acid sequence for aga $\mathrm{A}$ confirms the evidence that the Asp, which is responsible for side-chain interaction with $\mathrm{Ca}^{2+}$, is conserved among the GHF16 family members (1). Moreover, among the metal ions, which are mainly present in seawater $\left(\mathrm{Na}^{+}, \mathrm{Mg}^{2+}, \mathrm{K}^{+}\right.$and $\left.\mathrm{Ca}^{2+}\right)$, $\mathrm{K}^{+}$shows considerable effect on rAgaA activity, suggesting that the red algae is originating from the marine environment. It is possible that the reason that $\mathrm{Zn}^{2+}$ has an inhibitory effect on rAgaA might be the structural alteration of the enzyme due to the affinity of the heavy metals for the $\mathrm{SH}, \mathrm{CO}$ and $\mathrm{NH}$ moieties of the amino acids in the protein.

In conclusion, a marine bacterium Pseudoalteromonas sp. was isolated from red algae in the Jeju island (Korea) coastal environment. AgaA features homologous catalytic domains belonging to the GHF-16 family, and has characteristic properties indicating neoagarooligosaccharide production. Due to the reported functional properties of the neoagarooligosaccharides (25) when obtained from agar, the purified $\mathrm{r}$ rAgaA enzyme has potential for usage in industries for the production of pharmaceuticals and cosmetics. To further advance the research, the crystal structure and threedimensional structure analyses need to be carried out in order to understand the mechanism of the catalysis of rAgaA in detail, and thereby investigate the structure-function relationships of the protein. Since no primary structural and functional characteristics have been reported for Aeromonas $\beta$ agarases to date, the current investigation will be useful for studying the structure, function and evolution of Aeromonas $\beta$ agarase as well.

\section{ACKNOWLEDGEMENTS}

This research was financially supported by the Ministry of Education, Science Technology (MEST) and Korea Industrial Technology Foundation (KOTEF) through the Human Resource Training Project for Regional Innovation, and by a research grant (PE98472) from Korea Ocean Research \& Development Institute.

\section{REFERENCES}

1. Allouch, J.; Jam, M.; Helbert, W.; Barbeyron ,T.; Kloareg, B.; Henrissat, B.; Czjzek, M. (2003). The three-dimensional structures of two beta agarases. J. Biol. Chem. 278, 47171-47180.

2. Aoki, T.; Araki, T.; Kitamikado, M. (1990). Purification and characterization of a novel beta-agarase from Vibrio sp. AP-2. Eur. J. Biochem. 187, 461-465.

3. Araki, C. (1959). Seaweed polysaccharides. In: Wolfrom ML (ed.), Carbohydrate chemistry of substances of biological interests. Pergamon Press, London, pp 15-30.

4. Araki, C. (1966). Some recent studies on the polysaccharide of agarophytes. In: Young EG, Maclachan JL (ed.), Proceedings International seaweed symposium. 5, 1965. Pergamon Press, London, pp 3-17.

5. Araki, C.; Arai, K. (1967). Studies on the chemical constitution of Agaragar. XXIV. Isolation of a new disaccharide as a reversion product from acidic hydrolysate. Bull. Chem. Societ. Japan. 40, 1452-1456.

6. Araki, T.; Lu, Z.; Morishita, T. (1998). Optimization of parameters for isolation of protoplasts from Gracilaria verrucosa (Rhodophyta). J. Mar. 
Biotechnol. 6, 193-197.

7. Barbeyron, T.; Gerard, A., Potin, P.; Henrissat, B.; Kloareg, B. (1998). The kappa-carrageenase of the marine bacterium Cytophaga drobachiensis. Structural and phylogenetic relationships within family 16 glycoside hydrolases. Mol. Biol. Evol. 15, 528-537.

8. Beg, Q.K.; Gupta, R. (2003). Purification and characterization of an oxidation-stable. Thiol depedent serine alkaline protease from Bacillus mojavensis. Enzyme Microb. Technol. 32, 294-304.

9. Chen, H.M.; Zheng, L.; Yan, X. J. (2005). The preparation and bioactivity research of agaro-oligosaccharides. Food Technol. Biotechnol. 43, 29-36.

10. Davies, G.; Henrissat, B. (1995). Structures and mechanisms of glycosyl hydrolases. Structure. $15 ; 3(9), 853-859$.

11. Duckworth, M.; Turvey, J. R. (1969). The action of a bacterial agarase on agarose, porphyran and alkali treated porphyran. Biochem. J. 113, 687692.

12. Duckworth, M.; Yaphe, W. (1971). Structure of agar. I. Fractionation of complex mixture of polysaccharides. Carbohydr. Res. 16,189-197.

13. Ekborg, N.A.; Taylor, L.E.; Longmire, A.G.; Henrissat, B.; Weiner, R. M.; Hutcheson, S.W. (2006). Genomic and proteomic analyses of the agarolytic system expressed by Saccharophagus degradans 2-40. Appl. Environ. Microbiol. 72, 3396-3405.

14. Fu, W.; Han, B.; Duan, D., Liu, W., Wang, C. (2008a). Purification and characterization of agarases from a marine bacterium Vibrio sp. F-6. J. Indust. Microbiol. Biotechnol. 35, 915-922.

15. Fu, X.T.; Lin, H.; Kim, S.M. (2008b). Purification and characterization of a novel beta-agarase, AgaA34, from Agarivorans albus YKW-34. Appl. Microbiol. Biotechnol. 78, 265-273.

16. Groleau, D.; Yaphe, W. (1977). Enzymatic hydrolysis of agar: purification and characterization of beta-neoagarotetraose hydrolase from Pseudomonas atlantica. Can. J. Microbiol. 23, 672-679.

17. Ha, J.C.; Kim, J.T.; Kim, S.K.; Oh, T.K.; Yu, J.H; Kong, I.S. (1997). Beta agarase from Pseudomonas sp. W7: purification of the recombinant enzyme from Escherichia coli and the effects of salt on its activity. Biotechnol. Appl. Biochem. 26,1-6.

18. Haddar, A.; Bougatef, A.; Agrebi, R.; Sellami-Kamoun, A.; Nasri, M. (2009). A novel surfactant-stable alkaline serine protease from a newly isolated Bacillus mojavensis A21. Purification and characterization. Process. Biochem. 44, 29-35.

19. Hassairi, I.; Ben, A.R.; Nonus, M.; Gupta, B.B. (2001). Production and separation of alpha-agarase from Altermonas agarlyticus strain GJ1B. Biores. Technol. 79, 47-51.

20. Hu, Z.; Lin, B.K.; Xu, Y.; Zhong, M.Q.; Liu, G.M. (2009). Production and purification of agarase from a marine agarolytic bacterium Agarivorans sp. HZ105. J. Appl. Microb. 106, 181-190.

21. Jacobs, M.; Elkiasson, M.; Uhlen, M.; Flock, J.I. (1985). Cloning, sequencing and expression of subtilisin carlsberg from Bacillus licheniformis. Nucleic Acids Res. 13, 8913-8927.

22. Jam, M.; Flament, D.; Allouch, J.; Potin, P.; Thion, L.; Kloareg, B.;
Czjzek, M.; Helbert, W.; Michel, G.; Barbeyron. T. (2005). The endobeta-agarases AgaA and AgaB from the marine bacterium Zobellia galactanivorans: two paralogue enzymes with different molecular organizations and catalytic behaviours. Biochem. J. 385, 703-713.

23. Kendall, K.; Cullum, J. (1984). Cloning and expression of an extracellular-agarase from Streptomyces coelicolor A3(2) in Streptomyces lividans 66, Gene 29, 315-321.

24. Kirimura, K.; Masuda, N.; Iwasaki, Y.; Nakagawa, H.; Kobayashi, R.; Usami. S. (1999). Purification and characterization of a novel beta agarase from an alkalophilic bacterium, Alteromonas sp. E-1. J. Biosci. Bioeng. 87, 436-441.

25. Kobayashi, R., Takisada, M., Suzuki, T., Kirimura, K.; Usami, S. (1997). Neoagarobiose as a novel moisturizer with whitening effect. Biosci. Biotechnol. Biochem. 61, 162-163.

26. Lee, S.; Jang, D. J. (2001). Progressive rearrangement of subtilisin Carlsberg into orderly and inflexible conformation with $\mathrm{Ca}^{2+}$ binding. Byophys. J. 81, 2972-2978.

27. Lee, S.; Park, J.; Yoon, S.; Kim, J.; Kong, I. (2000). Sequence analysis of a beta-agarase gene (pjaA) from Pseudomonas sp. isolated from marine environment. J. Biosci. Bioeng. 89, 485-488.

28. Lee, D.G.; Park, G.T.; Kim, N.Y.; Lee, E.J.; Jang, M.K.; Shin, Y.G.; Park, G.S.; Kim, T.M.; Lee, J.W.; Lee, J.H.; Kim, S.J.; Lee, S.H. (2006). Cloning, expression, and characterization of a glycoside hydrolase family 50 beta-agarase from a marine Agarivorans isolate. Biotechnol. Lett. $28,1925-1932$.

29. Leon, O.; Quintana, L.; Peruzzo, G.; Slebe, J.C. (1992). Purification and properties of an extracellular agarase from Alteromonas sp. strain $\mathrm{C}-1$. Appl. Environ. Microb. 58, 4060-4063.

30. Ma, C.; Lu, X.; Shi, C.; Shi, C.; Li, J.; Gu, Y.; Ma, Y.; Chu, Y.; Han, F.; Gong, Q. (2007). Molecular cloning and characterization of a novel betaagarase, AgaB, from marine Pseudoalteromonas sp. CY24. J. Biol. Chem. 282, 3747-3754.

31. Michel, G.; Chantalat, L.; Duee, E.; Barbeyron, T.; Henrissat, B.; Kloareg, B.; Dideberg, O. (2001). The kappa-carrageenase of Pseudoalteromonas carrageenovora features a tunnel-shaped active site: a novel insight in the evolution of clan-B glycoside hydrolases Structure. 9, 513-525.

32. Miller, G.L. (1959). Use of dinitrosalicylic acid reagent for determination of reducing sugar. Anal. Chem. 31,426-428.

33. Morrice, L. M.; McLean, M.W.; Williamson, F.B.; Long, W.F. (1983). Beta-agarases I and II from Pseudomonas atlantica. Purifications and some properties. Eur. J. Biochem. 135, 553-558.

34. Ohta, Y.; Hatada, Y.; Ito, S.; Horikoshi, K. (2005). High-level expression of a neoagarobiose-producing beta-agarase gene from Agarivorans sp. JAMB-A11 in Bacillus subtilis and enzymic properties of the recombinant enzyme. Biotechnol. Appl. Biochem. 41,183-191.

35. Ohta, Y.; Hatada, Y.; Nogi, Y.; Li, Z.; Ito, S.; Horikoshi, K. (2004a). Cloning, expression, and characterization of a glycoside hydrolase family 86 beta agarase from a deep-sea Microbulbifer-like isolate. Appl. Microb. 
Biotechnol. 66, 266-275.

36. Ohta, Y.; Hatada, Y.; Nogi, Y.; Miyazaki, M.; Li, Z.; Akita, M.; Hidaka, Y.; Goda, S.; Ito, S.; Horikoshi, K. (2004b). Enzymatic properties and nucleotide and amino acid sequences of a thermostable beta-agarase from a novel species of deep-sea Microbulbifer. Appl. Microb. Biotechnol. 64, 505-514.

37. Ohta, Y.; Hatada, Y.; Nogi, Y.; Miyazaki, M.; Li, Z.; Hatada, Y.; Ito, S.; Horikoshi, K. (2004c). Enzymatic properties and nucleotide and amino acid sequences of a thermostable beta agarase from the novel marine isolate, JAMB-A94. Biosci. Biotechnol. Biochem. 68 (5), 1073-1081.

38. Pearson, W.R. (1990). Rapid and Sensitive Sequence Comparison with FASTP and FASTA. Methods Enzymol. 183, 63-98.

39. Potin, P.; Richard, C.; Rochas, C.; Kloareg, B. (1993). Purification and characterization of the alpha-agarase from Alteromonas agarlyticus (Cataldi) comb. nov., strain GJ1B. Eur. J. Biochem. 214, 599-607.

40. Pugsley, A.P. (1993). The complete general secretory pathway in gram negative bacteria. Microb. Rev. 57, 50-108.

41. Ryu, S.K.; Cho, S.J.; Park, S.R.; Lim, W.J.; Kim, M.K.; Hong, S.Y.; Baed, W.; Park, Y.W.; Kim, B.K.; Kim, H.; Yun, H.D. (2001). Cloning of the cel9A gene and characterization of its gene product from marine bacterium Pseudomonas sp. SK38. Appl. Microb. Biotechnol. 57, 138145 .

42. Sambrook, J.; Fritsch, E.F.; Maniatis. T. (1989). Molecular cloning: a laboratory manual, $2^{\text {nd }}$ edn. Cold Spring Harbor Laboratory, Cold Spring Harbor, N.Y.

43. Schroeder, D.C.; Jaffer, M.A.; Coyne, V.E. (2003). Investigation of the role of a beta (1-4) agarase produced by Pseudoalteromonas gracilis B9 in eliciting disease symptoms in the red alga Gracilaria gracilis. Microbiol. 149, 2919-2929.

44. Sugano, Y.; Kodama, H.; Terada, I.; Yamazaki, Y.; Noma, M. (1994). Purification and characterization of a novel enzyme, $\alpha$ neoagarooligosaccharide hydrolase ( $\alpha$-NAOS hydrolase), from a marine bacterium, Vibrio sp. strain JT0107. J. Bacteriol. 176, 6812-6818.

45. Sugano, Y.; Terada, I.; Arita, M.; Noma, M.; Matsumoto, T. (1993). Purification and characterization of a new agarase from a marine bacterium, Vibrio sp. strain JT0107. Appl. Environ. Microbiol. 59, 15491554.

46. Suzuki, H.; Sawai, Y.; Suzuki, T.; Kawai, K. (2003). Purification and characterization of an extracellular beta agarase from Bacillus sp. MK03. J. Biosci. Bioeng. 93, 456-463.

47. Van der Meulen, H.J.; Harder, W. (1975). Production and characterization of the agarase of Cytoplaga flevensis. Antonie van Leeuwenhoek. 41, 431-447.

48. Vera, J.; Alvarez, R.; Murano, E.; Slebe, J.C.; Leon, O. (1998). Identification of a marine agarolytic Pseudoalteromonas isolate and characterization of its extra cellular agarase. Appl. Environ. Microbiol. $64,4378-4383$.

49. Wang, J.; Mou, H.; Jiang, X.; Guan, H. (2006). Characterization of a novel beta-agarase from marine Alteromonas sp. SY37-12 and its degrading products. Appl. Microbiol. Biotechnol. 71, 833-839.

50. Zhang, W.W.; Sun, L. (2007). Cloning, characterization, and molecular application of a beta-agarase gene from Vibrio sp. strain V134. Appl. Environ. Microbiol. 73, 2825-2831. 